\title{
Molecular Characteristics and Antibiotic Resistance Profiles of Klebsiella Isolates in Mthatha, Eastern Cape Province, South Africa
}

\author{
Sandeep Vasaikar, ${ }^{1}$ Larry Obi, ${ }^{2}$ Isaac Morobe, ${ }^{3}$ and Mary Bisi-Johnson ${ }^{4}$ \\ ${ }^{1}$ Division of Medical Microbiology, Department of Laboratory Medicine and Pathology, Faculty of Health Sciences, \\ Walter Sisulu University, Private Bag X1, Mthatha, Eastern Cape 5117, South Africa \\ ${ }^{2}$ Academic Affairs, University of Fort Hare, Private Bag X1314, Alice 5700, South Africa \\ ${ }^{3}$ Department of Biological Sciences, University of Botswana, Mobutu Drive, P.O. Box 80462, Gaborone, Botswana \\ ${ }^{4}$ Department of Microbiology, Obafemi Awolowo University, Ile-Ife, Osun State, Nigeria
}

Correspondence should be addressed to Sandeep Vasaikar; sandeepvasaikar@yahoo.com

Received 15 July 2016; Accepted 27 December 2016; Published 30 January 2017

Academic Editor: Barbara H. Iglewski

Copyright (C) 2017 Sandeep Vasaikar et al. This is an open access article distributed under the Creative Commons Attribution License, which permits unrestricted use, distribution, and reproduction in any medium, provided the original work is properly cited.

\begin{abstract}
The increase in the incidence of extended-spectrum $\beta$-lactamase- (ESBL-) producing Klebsiella species has become a serious problem worldwide, because of their incrimination in antibiotic resistance. The objective of this study is to investigate the resistance genes responsible for ESBL-producing Klebsiella species and carbapenemase-producing Klebsiella (CRE) isolated in Mthatha and to study their epidemiology. A prospective, descriptive study of 202 nonrepetitive samples from patients was obtained from Nelson Mandela Academic Hospital. The cultured Klebsiella isolates were subjected to antimicrobial susceptibility tests and the polymerase chain reaction of $b l a_{\mathrm{CTX}-\mathrm{M}}, b l a_{\mathrm{TEM}}, b l a_{\mathrm{SHV}}, b l a_{\mathrm{KPC}}$, and $b l a_{\mathrm{NDM}}$ genes. Overall $\mathrm{K}$. pneumoniae were the majority with $169(83.7 \%)$ species isolates, followed by K. oxytoca with 29 (14.4\%), while K. ozaenae and Raoultella ornithinolytica were $2(0.9 \%)$ each. The prevalence of ESBL production in all Klebsiella species was 117 (57.9\%). ESBL-genotypic resistance is driven in Mthatha by bla $a_{\mathrm{SHV}}$ $121(77.1 \%)$ followed by $b l a_{\text {TEM }} 105(66.9 \%)$ and $b l a_{\text {CTX-M }}$ at $89(56.7 \%)$. The most common ESBL genotype combination among the Klebsiella was $b l a_{\mathrm{TEM}}+b l a_{\mathrm{SHV}}+b l a_{\mathrm{CTX- \textrm {M }}}$ at $79(50.3 \%)$. There is a steady increase in the rate of ESBL genes in the last five years.
\end{abstract}

\section{Introduction}

The genus Klebsiella belongs to the Enterobacteriaceae family and comprises Gram-negative opportunistic nonmotile pathogens with a mucoid aspect. The gastrointestinal tract serves as a reservoir and is often the latent source for infections [1]. The genus Klebsiella is classified into four species: Klebsiella pneumoniae (K. pneumoniae), Klebsiella oxytoca ( $K$. oxytoca), Klebsiella terrigena ( $K$. terrigena), and Klebsiella planticola (K. planticola), with K. pneumoniae consisting of three subspecies, $K$. pneumoniae subsp. pneumoniae, K. pneumoniae subsp. ozaenae, and K. pneumoniae subsp. rhinoscleromatis [2]. K. pneumoniae is an opportunistic microorganism which causes serious diseases such as septicemia, pneumonia, urinary tract infections (UTIs), chronic lung disorders, and nosocomial infections in immunocompromised patients [3]. The emergence of extended-spectrum $\beta$-lactamase- (ESBL-) producing bacteria, particularly in K. pneumoniae, is now a critical concern for the development of therapies against bacterial infection [4]. These strains are resistant to extended-spectrum betalactam antibiotics, aminoglycosides, and fluoroquinolones [5]. The currently dominant ESBLs belonging to class A in the Ambler classification are $b l a_{\mathrm{TEM}}, b l a_{\mathrm{SHV}}$, and $b l a_{\mathrm{CTX}-\mathrm{M}}$ types. The detection of the common ESBL genes such as $b l a_{\mathrm{TEM}}, b l a_{\mathrm{SHV}}$, and $b l a_{\mathrm{CTX}-\mathrm{M}}$ by molecular methods in the ESBL-producing bacteria and their patterns of antimicrobial resistance can provide useful information about their epidemiology and can aid in developing rational antimicrobial therapy. K. pneumoniae carbapenemase (KPC) are Ambler class A plasmid-encoded enzymes that are capable of hydrolyzing all beta-lactam antibiotics, including 
monobactams, extended-spectrum cephalosporins, and carbapenems. Originally described in 2001, pathogens harbouring these antibiotic resistance enzymes have been reported from the United States of America, France, China, Sweden, Norway, Colombia, and Brazil. Molecular detection of the $b l a_{\mathrm{KPC}}$ gene by polymerase chain reaction (PCR) assay provides laboratories with a means to quickly identify the presence of this important resistance determinant gene [6]. Though many studies have reported the drug resistance of $K$. pneumoniae and K. oxytoca worldwide [4, 7-9]. Epidemiology studies on ESBL-producing $K$. pneumoniae in Republic of South Africa (RSA) from different provinces have been reported [10-13], but little is known in the Eastern Cape Province (ECP) about the epidemiology and molecular characteristics of ESBLs. The aim of this study was to investigate the resistance mechanisms to among ESBL-producing different Klebsiella species and carbapenemase-producing Klebsiella (CRE) isolated in Mthatha and surrounding areas and to study antimicrobial susceptibility to parenteral and oral antimicrobials.

\section{Materials and Methods}

\subsection{Experimental Design}

2.1.1. Ethical Considerations. Ethical approval for the study was granted by the Health Research Ethics and Biosafety Committee of the Walter Sisulu University (WSU) certificate number 022/110 and the Nelson Mandela Academic Hospital Ethics Committee (NMAH), Mthatha, ECP.

2.1.2. Study Design and Setting. A prospective, descriptive study based on laboratory investigations at the Microbiology Laboratory of the National Health Laboratory Services (NHLS) at NMAH and the Department of Medical Microbiology, Faculty of Health Sciences, WSU was undertaken. In this study 203 nonrepetitive (one per patient) samples from patients were randomly obtained from August 2011 to May 2014. Figure 1 shows the specimen catchment area, that is, Mthatha and surrounding clinics. Mthatha (formerly Umtata) is the main town of the King Sabata Dalindyebo Local Municipality in the Oliver Reginald Tambo District of the ECP in South Africa. Study areas and health facilities in Mthatha and surrounding areas were primary health centres/clinics, secondary district hospitals, and a tertiary/academic hospital.

2.1.3. Specimens. Nonduplicate, randomly selected Klebsiella isolates were collected from Mthatha and surrounding-area clinics. Specimens included blood culture and catheter tips, swabs from abscesses, eye, ear, and vagina, sputum and throat swabs, urine, and sterile fluids (plural fluid, synovial fluid, etc.). Demographic data of the patients recorded were date of specimen collection, age, gender, specimen, tests ordered, and hospital/clinic and provisional diagnosis.

2.2. Microbiologic Methods. All samples were routinely cultured on MacConkey and blood agar plates. Blood and sputum were also cultured on chocolate agar. All suspected

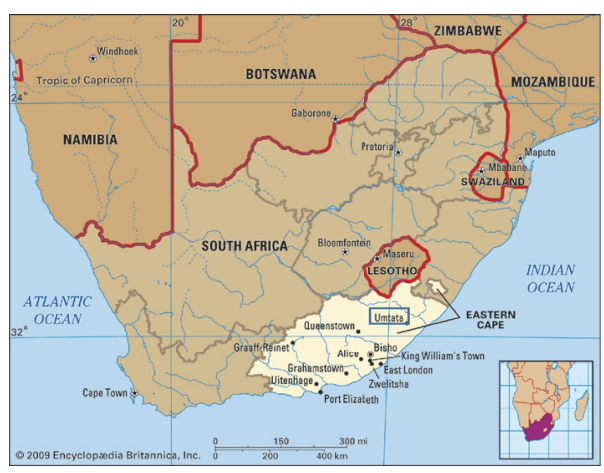

Figure 1: Map of South Africa showing study area, Umtata (now Mthatha), in the province of Eastern Cape (by courtesy of Encyclopaedia Britannica, Inc., copyright 2009; used with permission) [14].

colonies were identified by gram staining, colony characteristics, motility, and so forth. Strains were identified to the species level with bioMérieux API20E and confirmed by Siemens MicroScan Negative ID panel Type 2 . MICs were determined using MicroScan dehydrated broth microdilution panel negative MIC Type 37 (Siemens Medical Solutions Diagnostics, West Sacramento, CA), following the manufacturer's guidelines and Clinical Laboratory Standards Institute (CLSI) [15]. MICs were interpreted following CLSI guidelines, including the new clinical breakpoints published in 2010 for carbapenems [16]. ESBL detection: phenotypicthe ESBL detection was done as was recommended by the CLSI confirmatory procedure, by using cefotaxime $(30 \mu \mathrm{g})$ and ceftazidime $(30 \mu \mathrm{g})$ discs alone and in combination with clavulanic acid discs. K. pneumoniae (ATCC-700603) were used as the controls throughout the study [17]. The ESBL production was confirmed by MicroScan MIC 37 panel using combination of cefotaxime/K clavulanate (Cft/CA) and ceftazidime/K clavulanate (Caz/CA) [18].

\subsection{Molecular ESBL Detection by $r P C R$}

2.3.1. DNA Extraction. DNA extraction was done using Roche MagNA Pure Bacteria Lysis Buffer, MagNA Pure Compact Nucleic Acid Isolation Kit 1 in MagNA Pure Compact System (Roche Applied Science, Indianapolis).

2.3.2. Real-Time PCR for $b l a_{\mathrm{CTX}-\mathrm{M}}, b l a_{\mathrm{TEM}}, b l a_{\mathrm{SHV}}, b l a_{\mathrm{NDM}}$, and $b l a_{\mathrm{KPC}}$. Real-time polymerase chain reaction (rPCR) was carried out in the LightCycler 2.0 instrument (Roche Applied Science, Germany) using LightCycler 480 Probes Master kit (Roche Diagnostics, USA). The $b l a_{\mathrm{CTX}}, b l a_{\text {TEM }}$, and $b l a_{\mathrm{SHV}}$ for ESBL and $b l a_{\mathrm{NDM}}$ and $b l a_{\mathrm{KPC}}$ genes for CRE were amplified by singleplex rPCR using the primers shown in Table 1. Primers were designed by TIB-Molbiol (Berlin, Germany) based on primers used by Turton et al. [19]. rPCR assay was performed in a 32 carousels using $20 \mu \mathrm{L}$ capillaries with each capillary containing a total volume of $20 \mu \mathrm{L}$ including $2 \mu \mathrm{L}$ of LightCycler FastStart DNA Master HybProbe, $2 \mu \mathrm{L}$ of primers $(0.5 \mathrm{mM}$ for each forward and reverse), $2.4 \mu \mathrm{L}$ of $\mathrm{MgCl}_{2}, 2 \mu \mathrm{L}$ of extracted DNA, and water 
TABLE 1: Primer sequences used for $b l a_{\mathrm{CTX}-\mathrm{M}}, b l a_{\mathrm{TEM}}, b l a_{\mathrm{SHV}}, b l a_{\mathrm{KPC}}$, and $b l a_{\mathrm{NDM}}$ genes detection.

\begin{tabular}{|c|c|c|c|}
\hline Target gene & Primer sequences $\left(5^{\prime}-3^{\prime}\right)$ & $\mathrm{Tm}$ in ${ }^{\circ} \mathrm{C}$ & Target size bp \\
\hline CTX-M forward primer & ATG TGC AGY ACC AGT AAR GTK ATG GC & 58.7 & \multirow{6}{*}{$336 \mathrm{bp}$} \\
\hline CTX-M reverse primer & ATC ACK CGG RTC GCC NGG RAT & 59.3 & \\
\hline CTX-M1 Probe & FAM- CCC gAC AgC Tgg gAg ACg AAA CgT & 70.2 & \\
\hline CTX-M2 Probe (gr 2) & YAK- CAggTgCTTATCgCTCTCgCTCTgTT & 66.3 & \\
\hline CTX-M9 all/1 Probe & LC640- Cg AC AAT ACNgCC ATg AA & 41.0 & \\
\hline CTX-M9 Probe & LC610- CTggATCgCACTgAACCTACgCTgA & 53.0 & \\
\hline TEM forward primer & AAG TTC TGC TAT GTG CGG TA & 59.7 & \multirow{2}{*}{$180 \mathrm{bp}$} \\
\hline TEM reverse primer & TGT TAT CAC TCA TGG TTA TGG CAG C & 59.5 & \\
\hline SHV- forward primer & CAG GAT CTG GTG GAC TAC T & 57.2 & \multirow{5}{*}{$195 \mathrm{bp}$} \\
\hline SHV- Reverse primer & GTC AAG GCG GGT GAC GTT & 59.3 & \\
\hline SHV-A primer & AAG GCG GGT GAC GTT GTC & 59.3 & \\
\hline SHV - S primer & CCG GTC AGC GAA AAA CAC & 57.0 & \\
\hline SHV -Probe & Cy5- TCT GGC GCA AAA AGG CAG TCA -BBQ & 64.5 & \\
\hline NDM Forward Primer & GAC CGC CCA GAT CCT CAA & 57.5 & \multirow{3}{*}{$51 \mathrm{bp}$} \\
\hline NDM Reverse Primer & CGC GAC CGG CAG GTT & 60.2 & \\
\hline NDM Probe & FAM- TGG ATC AAG CA+GGA+GAT -BBQ & 48.8 & \\
\hline KPC forward Primer & GGC CGC CGT GCA ATA C & 58,6 & \multirow{3}{*}{$61 \mathrm{bp}$} \\
\hline KPC reverse Primer & GCC CAA CTC CTT CA & 59,6 & \\
\hline KPC probe & Cy5- TGA TAA CGC GCG CAA TTT GT -BBQ & 68.9 & \\
\hline
\end{tabular}

Relevant positive strains for Klebsiella used as positive control in rPCR: CTX-M (group 1)- E. coli ATCC 25922, CTX-M (all groups except group 1) CAP2006D17*- TEM- K. pneumoniae ATCC 51503, SHV- K. pneumoniae ATCC 700603, KPC- K. pneumoniae ATCC BAA 1705, NDM- K. pneumoniae ATCC BAA 21246. Obtained from NICD (National Institute of Communicable Diseases, Johannesburg, South Africa).

to make up the volume of $20 \mu \mathrm{L}$. DNA amplification was carried out using preincubation step at $95^{\circ} \mathrm{C}$ for $30 \mathrm{~s}$, followed by 45 cycles of amplification with denaturation at $95^{\circ} \mathrm{C}$ for $30 \mathrm{~s}$, annealing and extension at $60^{\circ} \mathrm{C}$ for 1 minute without the third step, and then a single cycle of cooling. Absolute quantification was carried out using the LightCycler software 4.05 .

2.4. Statistical Analysis. Our data was entered into a database using SPSS 23 for Windows (SPSS Inc., Chicago, IL). The primary analysis involved using chi-square test in order to look for association between dependable variable (ESBL production) with covariable, for example, type of hospital/clinic, gender, and type of specimens. Significant variables were included in binary logistical regression to calculate the odds ratio and 95\% confidence interval. All statistical testing was two tailed and statistical significance was defined as $\leq 0.05$. Comparison of ESBL and non-ESBL groups was done using Epidat 3.1 software.

\section{Results}

During the study period, a total of 202 Klebsiella species were isolated from a range of clinical specimens of patients hospitalized in various wards of NMAH in Mthatha and surrounding areas with secondary hospitals and clinics. $K$. pneumoniae were the majority 169 (83.7\%) species isolated followed by K. oxytoca $29(14.4 \%)$ while $K$. ozaenae and $R$. ornithinolytica were $2(0.9 \%)$. The prevalence of ESBL production in all Klebsiella species was 122 (60.4\%) while ESBLproducing $K$. pneumoniae were 117 (69.2\%) followed by $K$. oxytoca $5(17.9 \%)$ and both K. ozaenae and R. ornithinolytica were ESBL negative. Female population was slightly more 106 (52.5\%) than males 96 (47.5\%).

In the multivariate analysis (backward logistic regression), using age group from day 1 to 5 years and tertiary level of hospitalization were found to be independent risk factors for infection due to ESBL Klebsiella species (Table 2).

High antibiotic resistance in decreasing order was observed in amp/sulbactam, mezlocillin 167 (82.7\%), piperacillin 160 (79.2\%), trimeth/sulfa 143 (70.8\%), cefazolin 139 (68.8\%), cefepime 130 (64.4\%), cefuroxime 129 (63.9\%), cefpodoxime $127(62.9 \%)$, aztreonam $126(62.4 \%)$, ceftazidime 124 (61.4\%), and tobramycin 108 (53.5\%).

Tables 4 and 5 show rPCR data, $b l a_{\mathrm{CTX}-\mathrm{M}}, b l a_{\mathrm{TEM}}, b l a_{\mathrm{SHV}}$, $b l a_{\mathrm{NDM}}$, and $b l a_{\mathrm{KPC}}$ rPCR, and LightCycler 2.0 results; realtime PCR was done on 157 specimens.

rPCR was done on 157 specimens which were 103 (65.6\%) of ESBL positive $K$. pneumoniae and $K$. oxytoca and 54 (34.4\%) ESBL negative $K$. pneumoniae, K. oxytoca, K. ozaenae, and $R$. ornithinolytica. In rPCR the most common genotype was bla $a_{\mathrm{SHV}} 121(77.1 \%)$ followed by bla $a_{\mathrm{TEM}} 105$ $(66.9 \%)$ and $b l a_{\text {CTX-M }}$ at $89(56.7 \%)$ as last.

As seen in Table 5 there is increase trend of ESBL genotypes over four years of study from 2011 to 2014 except in year 2013. The carbapenemase genes $b l a_{\mathrm{NDM}}$ and $b l a_{\mathrm{KPC}}$ were not detected.

\section{Discussion}

The emergence of plasmid mediated ESBLs among the members of Klebsiella has increased worldwide. The incidence of 
TABLE 2: Multivariate analysis of risk factors for ESBL-producing and nonproducing Klebsiella species in Mthatha and surrounding areas.

(a)

\begin{tabular}{lccc}
\hline Quantitative variable & $\begin{array}{c}\text { ESBL } \\
\text { Mean } \pm \text { SD }\end{array}$ & $\begin{array}{c}\text { NIL } \\
\text { Mean } \pm \text { SD }\end{array}$ & OR (95\% CI) \\
\hline Age (groups 0-5) & $22.78(22.77)$ & $30.9(22.7)$ & $2.32(1.20-4.52)$ \\
\hline
\end{tabular}

(b)

\begin{tabular}{lccc}
\hline Variable & $\begin{array}{c}\text { ESBL } \\
\text { Number (\%) }\end{array}$ & $\begin{array}{c}\text { NIL } \\
\text { Number (\%) }\end{array}$ & OR (95\% CI) \\
\hline Gender & $59(60.8)$ & $38(39.2)$ & \\
$\quad$ Male & $58(55.2)$ & $47(44.8)$ & $1.18(0.68-2.06)$ \\
Female & & & \\
Type of hospital & $3(21.4)$ & $11(78.6)$ & - \\
$\quad$ Primary* & $41(47.1)$ & $46(52.9)$ & $2.06(0.76-5.57)$ \\
Secondary & $73(72.3)$ & $28(27.7)$ & $5.96(2.21-16.03)$ \\
Tertiary & & & 0.153 \\
\hline
\end{tabular}

${ }^{*}$ Reported as resistant due to ESBL production.

ESBLs in the different parts of South Africa has been reported from $36.1 \%$ to be as high as $68.3 \%$ (Gauteng, Western Cape, KwaZulu-Natal, Free State, and Limpopo) [11-13]. ESBL in K. pneumoniae showed rate of $62 \%$ from seven public sector hospitals in 2010 [20]. We reported 57.9\% (117) ESBL producers, which is less than the above-mentioned South African provinces. In all of the above-mentioned studies ECP was not represented; this could be reason for slightly less rate of ESBLs in this area. In Africa ESBLs rate in different countries have increased from 11.7 to $77.8 \%$ among K. pneumoniae (study periods: 1999-2005 and 2010) [7, 21]. Our ESBL rate of $57.9 \%$ is within this range, but it is on higher side. It was interesting to note that specific ESBLs appeared to be unique to a certain country or region. Though the prevalence of ESBLs has been recognized in various parts of the country, there is only limited data on its genotypes in this part of South Africa.

High antibiotic resistance in decreasing order was observed in penicillins, cephalosporins, folate pathway inhibitors, monobactams, and aminoglycosides. Percentage of low resistance was seen in carbapenems, aminoglycosides (only amikacin), glycylcyclines (tigecycline), cephamycins (cefoxitin), quinolone (levofloxacin), phosphonic acids (fosfomycin), antipseudomonal penicillins $+\beta$-lactamase inhibitor (pip/tazo), and fluoroquinolones (ciprofloxacin) which can be considered for treatment of Klebsiella species in Mthatha. Although resistance of $K$. pneumoniae to ceftazidime is a useful marker of presence of ESBLs, some types of ESBL-producing organisms appear susceptible to ceftazidime according to standard methods, and ceftazidime resistance may be due to mechanisms other than ESBL production. We detected $61.4 \%$ resistance to ceftazidime by MIC method while $57.9 \%$ (117) Klebsiella were ESBL producers by the CLSI confirmatory test which correlates well. Antibiotic resistance to cefotaxime, ceftazidime, and cefepime was $125(61.9 \%)$, $124(61.4 \%)$, and 130 (64.4\%), respectively, which is almost identical. This suggests that there are multiple copies of several ESBL genes in the sample, which is supported by the genotypic results in which $66.2 \%$ specimens had two or more ESBL genes (Table 3). We observed that nearly twothirds of the Klebsiella ESBL isolates were also resistant to at least 2 useful non- $\beta$ lactam antibiotics used to treat UTI, such as ciprofloxacin, trimethoprim-sulfamethoxazole, and gentamicin. Similar observations have been made by other investigators [22].

In this study the most common genotype was $b l a_{\mathrm{SHV}}$ $118(75.2 \%)$ followed by $b l a_{\text {TEM }} 99(63.1 \%)$ and bla $a_{\text {CTX-M }} 89$ $(56.7 \%)$ as last. These genotypes were found in majority of ESBL positive Klebsiella species as compared to ESBL negative Klebsiella. Our study finding is similar to the study done from Italy in which $b l a_{\mathrm{SHV}}$ was found to be the dominant ESBL genotype and also from Spain [22,23]. ESBL genotype varies in different parts of the world; $b l a_{\text {CTX-M }}$ is the most prevalent gene in high proportion of samples from different parts of the world and also Morocco $[7,9]$. In Africa bla $a_{\mathrm{CTX}-\mathrm{M} 15}$ is the most prevalent gene in a high proportion of the samples, disregarding country. It is usually combined with other types of $b l a_{\mathrm{CTX}-\mathrm{M}}, b l a_{\mathrm{TEM}}$, and $b l a_{\mathrm{SHV}}$ genes [7]. In other parts of the world, for example, Canada [8] and Turkey [24], bla $a_{\mathrm{TEM}}$ was the most common ESBL genotype. The most common ESBL genotype combination among the Klebsiella species (especially in K. pneumoniae) was combination of $b l a_{\mathrm{TEM}}$ $+b l a_{\mathrm{SHV}}+b l a_{\mathrm{CTX}-\mathrm{M}}$ which is similar to a previous report from South Africa [12]. The second most common genotype combination was the combination of $b l a_{\mathrm{TEM}}+b l a_{\mathrm{SHV}}$ which is similar to the study from Japan [4]. We found increased trend of Klebsiella ESBL genotypes over 4 years; this trend is seen in studies from different parts of the world, Japan and Canada $[4,8]$.

CRE has become an international health issue and poses a major threat to the viability of currently available antibiotics. First KPC was reported from South Africa in August 2011 from private hospital in Gauteng [25]. In our study we observed resistance to carbapenems by phenotypic test as ertapenem $3.5 \%$ while meropenem and imipenem were all susceptible. But all specimens were negative for $b l a_{\mathrm{KPC}}$ 
TABLE 3: Percentage of resistance (included intermediate resistance for statistical analysis) in ESBL and non-ESBL Klebsiella $(N=202)$.

\begin{tabular}{|c|c|c|c|c|c|}
\hline \multirow{2}{*}{ Antibiotic } & Total $(n=202)$ & $\operatorname{ESBL}(n=117)$ & NIL $(n=85)$ & \multirow{2}{*}{ OR (95\% CI) } & \multirow{2}{*}{ Pvalue ${ }^{\mathrm{a}}$} \\
\hline & Number (\%) & Number (\%) & Number (\%) & & \\
\hline Amikacin & $10(5.0)$ & $5(4.3)$ & $5(5.9)$ & $0.71(0.20-2.55)$ & 0,604 \\
\hline Amox/K clav & $111(55.0)$ & $98(83.3)$ & $13(15.3)$ & $28.6(13.25-61.59)$ & 0,000 \\
\hline Ampicillin & $137(67.8)$ & $115(98.3)$ & $22(25.9)$ & $162.0(36.88-711.93)$ & 0,000 \\
\hline Amp/sulbactam* & $201(99.5)$ & $117(100.0)$ & $84(98.8)$ & - & 1,000 \\
\hline Aztreonam* & $126(62.4)$ & $117(100.0)$ & $9(10.6)$ & - & 0,996 \\
\hline Cefazolin* & $139(68.8)$ & $117(100.0)$ & $22(25.9)$ & - & 0,996 \\
\hline Cefepime* & $130(64.4)$ & $117(100.0)$ & $13(15.3)$ & - & 0,996 \\
\hline Cefotaxime & $124(61.4)$ & $117(100.0)$ & $7(8.2)$ & 116.5 (136.9-9105.9) & 0,000 \\
\hline Cefoxitin & $18(8.9)$ & $10(8.5)$ & $8(9.4)$ & $0.90(0.34-2.38)$ & 0,831 \\
\hline Cefpodoxime* & $127(62.9)$ & $117(100.0)$ & $10(11.8)$ & - & 0,996 \\
\hline Ceftazidime* & $124(61.4)$ & $117(100.0)$ & $7(8.2)$ & - & 0,996 \\
\hline Cefuroxime & $129(63.9)$ & $117(99.1)$ & $13(15.3)$ & $642.5(82.29-5016.08)$ & 0,000 \\
\hline Chloramphenicol & $71(35.1)$ & $53(45.3)$ & $18(21.2)$ & $3.25(1.71-6.19)$ & 0,000 \\
\hline Ciprofloxacin & $60(29.7)$ & $55(47.0)$ & $5(5.9)$ & $14.19(5.36-37.58)$ & 0,000 \\
\hline Ertapenem & $7(3.5)$ & $4(3.4)$ & $3(3.5)$ & $0.97(0.21-4.44)$ & 0,966 \\
\hline Fosfomycin & $26(12.9)$ & $15(12.8)$ & $11(12.9)$ & $0.97(0.42-2.25)$ & 0,954 \\
\hline Gentamicin & $103(51.0)$ & $92(78.6)$ & $11(12.9)$ & $24.76(11.44-53.60)$ & 0,000 \\
\hline Imipenem & $0(0.0)$ & $0(0.0)$ & $0(0.0)$ & - & - \\
\hline Levofloxacin & $23(11.4)$ & $19(16.2)$ & $4(4.7)$ & $3.88(1.27-11.86)$ & 0,018 \\
\hline Meropenem & $0(0.0)$ & $0(0.0)$ & $0(0.0)$ & - & - \\
\hline Mezlocillin* & $167(82.7)$ & $117(100.0)$ & $50(58.8)$ & - & 0,997 \\
\hline Moxifloxacin & $77(38.1)$ & $65(55.6)$ & $12(14.1)$ & $8.43(4.10-17.32)$ & 0,000 \\
\hline Nitrofurantoin $^{\Phi}$ & $1(0.5)$ & $0(0.0)$ & $1(1.2)$ & - & 1,000 \\
\hline Norfloxacin $^{\Phi}$ & $3(1.5)$ & $3(2.6)$ & $0(0.0)$ & - & 0,999 \\
\hline Pip/tazo & $31(15.3)$ & $28(23.9)$ & $3(3.5)$ & $8.60(2.52-29.36)$ & 0,001 \\
\hline Piperacillin* & $160(79.2)$ & $117(100.0)$ & $43(50.6)$ & - & 0,997 \\
\hline Tetracycline & $57(28.2)$ & $44(37.6)$ & $13(15.3)$ & $3.29(1.64-6.63)$ & 0,001 \\
\hline Tigecycline & $17(8.4)$ & $9(7.7)$ & $8(9.4)$ & $0.80(0.30-2.17)$ & 0,664 \\
\hline Tobramycin & $108(53.5)$ & $102(87.2)$ & $6(7.1)$ & $88.40(32.79-238.28)$ & 0,000 \\
\hline Trimeth/sulfa & $143(70.8)$ & $115(98.3)$ & $28(32.9)$ & $117.05(26.93-508.72)$ & 0,000 \\
\hline
\end{tabular}

${ }^{a}$ Exact $P$ values were determined by the $\chi^{2}$ test. For statistical analysis, the response of isolates to antibiotics was categorized as susceptible and nonsusceptible (consisting of intermediate and resistant groups). A $P$ value $<0.05$ was considered statistically significant. ${ }^{*}$ Reported as resistance due to ESBL production according to confirmation by Autoscan MIC37 panel. $\Phi$ : used only in urinary tract infections.

and $b l a_{\mathrm{NDM}}$ by genotypic test; this could be due to other resistance mechanisms such as newer CRE genes Verona Integron-Mediated Metallo- $\beta$-Lactamase (VIM), Imipenemase Metallo-Beta-Lactamase (IMP), or class D OXA-type enzymes [26].

In multivariate analysis we found age group 1-5 years (OR 2.32 [CI 1.20-4.52]) and tertiary health centre (OR 5.96 [CI 2.21-16.03]) were risk factors for developing ESBL. In ESBLs we found statistically significant $(P<0.005)$ antibiotic resistance in amox/clav, ampicillin, cefotaxime, cefuroxime, chloramphenicol, ciprofloxacin, gentamicin, levofloxacin, moxifloxacin, pip/tazo, tetracycline, tobramycin, and trimeth/sulfa.

\section{Conclusion}

This is the first report of molecular characteristics and antibiotic resistance profiles of Klebsiella isolates from clinical samples of patients in Mthatha, ECP, South Africa. The majority of Klebsiella species in our area are K. pneumoniae followed by $K$. oxytoca and less than $1 \%$ of $K$. ozaenae and $R$. ornithinolytica. This study reveals high ESBL rate in Klebsiella species especially in K. pneumoniae in this area. We also identified ESBL-producing three genes of $b l a_{\mathrm{TEM}}$, $b l a_{\mathrm{SHV}}$, and $b l a_{\mathrm{CTX}-\mathrm{M}}$ by $\mathrm{rPCR}$ and our antibiotic resistance in Klebsiella species in Mthatha which is driven by combination of $b l a_{\mathrm{TEM}}+b l a_{\mathrm{SHV}}+b l a_{\mathrm{CTX}-\mathrm{M}}$ and the most common genotype was $b l a_{\mathrm{SHV}}$ followed by $b l a_{\mathrm{TEM}}$ and $b l a_{\mathrm{CTX}-\mathrm{M}}$ as last. For treatment of drug resistant Klebsiella choice of antibiotics in decreasing order is carbapenems, amikacin, tigecycline, cefoxitin, levofloxacin, pip/tazo, ciprofloxacin, and fosfomycin for UTI. Fortunately we did not detect CREforming Klebsiella in Mthatha. There is steady increase in rate of ESBL genes in the last five years; therefore continuous surveillance is essential to monitor the ESBL-producing Klebsiella in hospitals and community and also for CRE. 
TABLE 4: Extended spectrum $\beta$-lactamase (ESBL) genotypes in Klebsiella strains.

\begin{tabular}{|c|c|c|c|c|}
\hline \multirow[b]{2}{*}{ Positive by PCR for ESBL genes } & \multicolumn{3}{|c|}{ Number amplified } & \multirow[b]{2}{*}{$\begin{array}{c}\text { Total } \\
(N=157)\end{array}$} \\
\hline & $\begin{array}{l}\text { K. pneumoniae } \\
\quad(n=139)\end{array}$ & $\begin{array}{c}\text { K. oxytoca } \\
(n=16)\end{array}$ & $\begin{array}{l}\text { R. ornitholytica and K. ozaenae } \\
\qquad(n=2)\end{array}$ & \\
\hline (A) Single ESBL gene & 28 & 2 & 2 & $32(20.4 \%)$ \\
\hline$b l a_{\mathrm{TEM}}$ only & 5 & 2 & 1 & $8(5.1 \%)$ \\
\hline$b l a_{\mathrm{SHV}}$ only & 22 & 0 & 1 & $23(14.7 \%)$ \\
\hline$b l a_{\mathrm{CTX}-\mathrm{M}}$ only & 1 & 0 & 0 & $1(0.6 \%)$ \\
\hline$b l a_{\mathrm{KPC}}$ only ${ }^{*}$ & 0 & 0 & 0 & 0 \\
\hline$b l a_{\mathrm{NDM}}$ only $^{*}$ & 0 & 0 & 0 & 0 \\
\hline (B) Two or more ESBL genes & 100 & 3 & 0 & $\begin{array}{c}103 \\
(65.6 \%)\end{array}$ \\
\hline$b l a_{\mathrm{CTX}-\mathrm{M}}+b l a_{\mathrm{TEM}}$ & 4 & 0 & 0 & $4(2.6 \%)$ \\
\hline$b l a_{\mathrm{CTX}-\mathrm{M}}+b l a_{\mathrm{SHV}}$ & 6 & 0 & 0 & $6(3.8 \%)$ \\
\hline$b l a_{\mathrm{TEM}}+b l a_{\mathrm{SHV}}$ & 12 & 2 & 0 & $14(8.9 \%)$ \\
\hline$b l a_{\mathrm{TEM}}+b l a_{\mathrm{SHV}}+b l a_{\mathrm{CTX}-\mathrm{M}}$ & 78 & 1 & 0 & $79(50.3 \%)$ \\
\hline$b l a_{\mathrm{KPC}}+b l a_{\mathrm{NDM}}^{*}$ & 0 & 0 & 0 & 0 \\
\hline
\end{tabular}

${ }^{*}$ Done in 52 selective ESBL positive isolates.

TABLE 5: Genotypes of Klebsiella bla $a_{\mathrm{TEM}}, b l a_{\mathrm{SHV}}$, and $b l a_{\mathrm{CTX}-\mathrm{M}}$ from 2011 to 2014.

\begin{tabular}{|c|c|c|c|c|c|}
\hline Genotypes & 2011 & 2012 & 2013 & 2014 & $\begin{array}{c}\text { Total } \\
(N=157)\end{array}$ \\
\hline$b l a_{\text {TEM }}$ positive alone or in combination & $23 / 34(67.7 \%)$ & $63 / 98(67.3 \%)$ & $6 / 8(75 \%)$ & $13 / 17(76.5 \%)$ & $105(66.9 \%)$ \\
\hline$b l a_{\mathrm{SHV}}$ positive alone or in combination & $19 / 34(55.9 \%)$ & $79 / 98(80.6 \%)$ & $8 / 8(100 \%)$ & $15 / 17(88.2 \%)$ & $121(77.1 \%)$ \\
\hline$b l a_{\text {CTX-M }}$ positive alone or in combination & $17 / 34(50 \%)$ & $54 / 98(55.1 \%)$ & $6 / 8(75 \%)$ & $12 / 17(70.6 \%)$ & $89(56.7 \%)$ \\
\hline
\end{tabular}

\section{Competing Interests}

The authors declare that they have no conflict of interests.

\section{Acknowledgments}

This work was fully supported by the WSU Institutional Research Grant 2013, 2014, and 2015. One of the authors is thankful to his Ph.D. supervisors-Professor Obi and Professor Banach-for their guidance; the Research Department WSU for funding of the study from the Institutional Research Grant; Nelson Mandela Academic Hospital and NHLSMthatha for providing patients and isolates; Dr. Pulido for help with statistical analysis; and Barbara for English language editing.

\section{References}

[1] R. El Fertas-Aissani, Y. Messai, S. Alouache, and R. Bakour, "Virulence profiles and antibiotic susceptibility patterns of Klebsiella pneumoniae strains isolated from different clinical specimens," Pathologie Biologie, vol. 61, no. 5, pp. 209-216, 2013.

[2] X. Li, D. Zhang, F. Chen, J. Ma, Y. Dong, and L. Zhang, "Klebsiella singaporensis sp. nov., a novel isomaltulose-producing bacterium," International Journal of Systematic and Evolutionary Microbiology, vol. 54, no. 6, pp. 2131-2136, 2004.

[3] H. Amraie, P. Shakib, S. Rouhi, N. Bakhshandeh, and B. Zamanzad, "Prevalence assessment of magA gene and antimicrobial susceptibility of Klebsiella pneumoniae isolated from clinical specimens in Shahrekord, Iran," Iranian Journal of Microbiology, vol. 6, no. 5, pp. 311-316, 2014.

[4] Y. Harada, Y. Morinaga, K. Yamada et al., "Clinical and molecular epidemiology of extended-spectrum $\beta$-lactamaseproducing Klebsiella pneumoniae and Escherichia coli in a Japanese Tertiary Hospita," Journal of Medical Microbiology \& Diagnosis, vol. 2, no. 3, pp. 2161-2170, 2013.

[5] J. Du, P. Li, H. Liu, D. Lü, H. Liang, and Y. Dou, "Phenotypic and molecular characterization of multidrug resistant Klebsiella pneumoniae isolated from a University Teaching Hospital, China," PLoS ONE, vol. 9, no. 4, Article ID e95181, 2014.

[6] L. Metwally, N. Gomaa, M. Attallah, and N. Kamel, "High prevalence of Klebsiella pneumoniae carbapenemase-mediated resistance in K. pneumoniae isolates from Egypt," Eastern Mediterranean Health Journal, vol. 19, no. 11, pp. 947-952, 2013.

[7] V. Storberg, "ESBL-producing Enterobacteriaceae in Africa-a non-systematic literature review of research published 20082012," Infection Ecology \& Epidemiology, vol. 4, 2014.

[8] A. J. Denisuik, P. R. S. Lagacé-Wiens, J. D. Pitout et al., "Molecular epidemiology of extended-spectrum $\beta$-lactamase-, AmpC $\beta$-lactamase- and carbapenemase-producing Escherichia coli and Klebsiella pneumoniae isolated from Canadian hospitals over a 5 year period: CANWARD 2007-11," The Journal of Antimicrobial Chemotherapy, vol. 68, supplement 1, pp. i57-i65, 2013.

[9] A. Barguigua, K. Zerouali, K. Katfy, F. El Otmani, M. Timinouni, and N. Elmdaghri, "Occurrence of OXA-48 and NDM-1 
carbapenemase-producing Klebsiella pneumoniae in a Moroccan university hospital in Casablanca, Morocco," Infection, Genetics and Evolution, vol. 31, pp. 142-148, 2015.

[10] J. D. D. Pitout, K. S. Thomson, N. D. Hanson, A. F. Ehrhardt, E. S. Moland, and C. C. Sanders, " $\beta$-Lactamases responsible for resistance to expanded-spectrum cephalosporins in Klebsiella pneumoniae, Escherichia coli, and Proteus mirabilis isolates recovered in South Africa," Antimicrobial Agents and Chemotherapy, vol. 42, no. 6, pp. 1350-1354, 1998.

[11] O. Perovic, A. Singh-Moodley, A. Dusé et al., "National sentinel site surveillance for antimicrobial resistance in Klebsiella pneumoniae isolates in South Africa, 2010-2012," South African Medical Journal, vol. 104, no. 8, pp. 563-568, 2014.

[12] L. Meyer, C. D. J. Labuschagne, M. M. Ehlers, M. G. Dove, and G. F. Weldhagen, "Diversity of bla-type genes in extendedspectrum $\beta$-lactamase-producing Klebsiella pneumoniae isolated during 2003-2004 at Pretoria Academic Hospital," South African Journal of Epidemiology and Infection, vol. 22, no. 1, pp. 5-7, 2007.

[13] J. M. Bell, J. D. Turnidge, A. C. Gales, M. A. Pfaller, and R. N. Jones, "Prevalence of extended spectrum $\beta$-lactamase (ESBL)-producing clinical isolates in the Asia-Pacific region and South Africa: regional results from SENTRY Antimicrobial Surveillance Program (1998-99)," Diagnostic Microbiology and Infectious Disease, vol. 42, no. 3, pp. 193-198, 2002.

[14] Encyclopaedia Britannica, Map of South Africa showing study area, Umtata (Now Mthatha) in the province of Eastern Cape, 2009, http://kids.britannica.com/elementary/art-181191/.

[15] Clinical and Laboratory Standards Institute, Methods for Dilution Antimicrobial Susceptibility Tests for Bacteria That Grow Aerobically, Approved Standard M7-A7, CLSI, Wayne, Pa, USA, 7 th edition, 2008.

[16] Clinical and Laboratory Standards Institute, Performance Standards for Antimicrobial Disk Susceptibility Tests, Approved Standard-M02-A11, vol. 32, no. 1 Replaces M02-A10, vol. 29, no. 1, CLSI, Wayne, Pa, USA, 11th edition, 2010.

[17] Clinical and Laboratory Standards Institute, Performance Standards for Antimicrobial Susceptibility Testing-Eighteenth Informational Supplement. Screening and Confirmatory Tests for ESBLs in Klebsiella pneumoniae, K. oxytoca, Escherichia coli and Proteus Mirabilis-Eighteenth Informational Supplement M100S18, vol. 28, no. 1, CLSI, Wayne, Pa, USA, 2008.

[18] M. Hombach, B. Mouttet, and G. V. Bloemberg, "Consequences of revised CLSI and EUCAST guidelines for antibiotic susceptibility patterns of ESBL- and AmpC $\beta$-lactamase-producing clinical Enterobacteriaceae isolates," Journal of Antimicrobial Chemotherapy, vol. 68, no. 9, pp. 2092-2098, 2013.

[19] J. F. Turton, H. Baklan, L. K. Siu, M. E. Kaufmann, and T. L. Pitt, "Evaluation of a multiplex PCR for detection of serotypes $\mathrm{K} 1, \mathrm{~K} 2$ and $\mathrm{K} 5$ in Klebsiella sp. and comparison of isolates within these serotypes," FEMS Microbiology Letters, vol. 284, no. 2, pp. 247-252, 2008.

[20] C. Bamford, K. Bonorchis, A. Ryan et al., "Antimicrobial susceptibility patterns of selected bacteraemic isolates from South African public sector hospitals, 2010," Southern African Journal of Epidemiology and Infection, vol. 26, no. 4, pp. 243250, 2011.

[21] G. S. Tansarli, P. Poulikakos, A. Kapaskelis, and M. E. Falagas, "Proportion of extended-spectrum $\beta$-lactamase (ESBL)producing isolates among Enterobacteriaceae in Africa: evaluation of the evidence-systematic review," Journal of Antimicrobial Chemotherapy, vol. 69, no. 5, pp. 1177-1184, 2014.
[22] N. Dayan, H. Dabbah, I. Weissman, I. Aga, L. Even, and D. Glikman, "Urinary tract infections caused by community-acquired extended-spectrum $\beta$-lactamase-producing and nonproducing bacteria: A Comparative Study," Journal of Pediatrics, vol. 163, no. 5, pp. 1417-1421, 2013.

[23] M. Perilli, E. Dell'Amico, B. Segatore et al., "Molecular characterization of extended-spectrum $\beta$-lactamases produced by nosocomial isolates of Enterobacteriaceae from an Italian nationwide survey," Journal of Clinical Microbiology, vol. 40, no. 2, pp. 611-614, 2002.

[24] E. B. Bali, L. Açik, and N. Sultan, "Phenotypic and molecular characterization of SHV, TEM, CTX-M and extended-spectrum $\beta$-lactamase produced by Escherichia coli, Acinobacter baumannii and Klebsiella isolates in a Turkish hospital," African Journal of Microbiology Research, vol. 4, no. 8, pp. 650-654, 2010.

[25] A. Brink, C. Feldman, G. Richards, J. Moolman, and M. Senekal, "Emergence of extensive drug resistance (XDR) among Gramnegative bacilli in South Africa looms nearer," South African Medical Journal, vol. 98, no. 8, pp. 586-592, 2008.

[26] T. Netikul and P. Kiratisin, "Genetic characterization of carbapenem-resistant enterobacteriaceae and the spread of carbapenem-resistant klebsiella pneumonia ST340 at a university hospital in Thailand," PLoS ONE, vol. 10, no. 9, Article ID e0139116, 2015. 

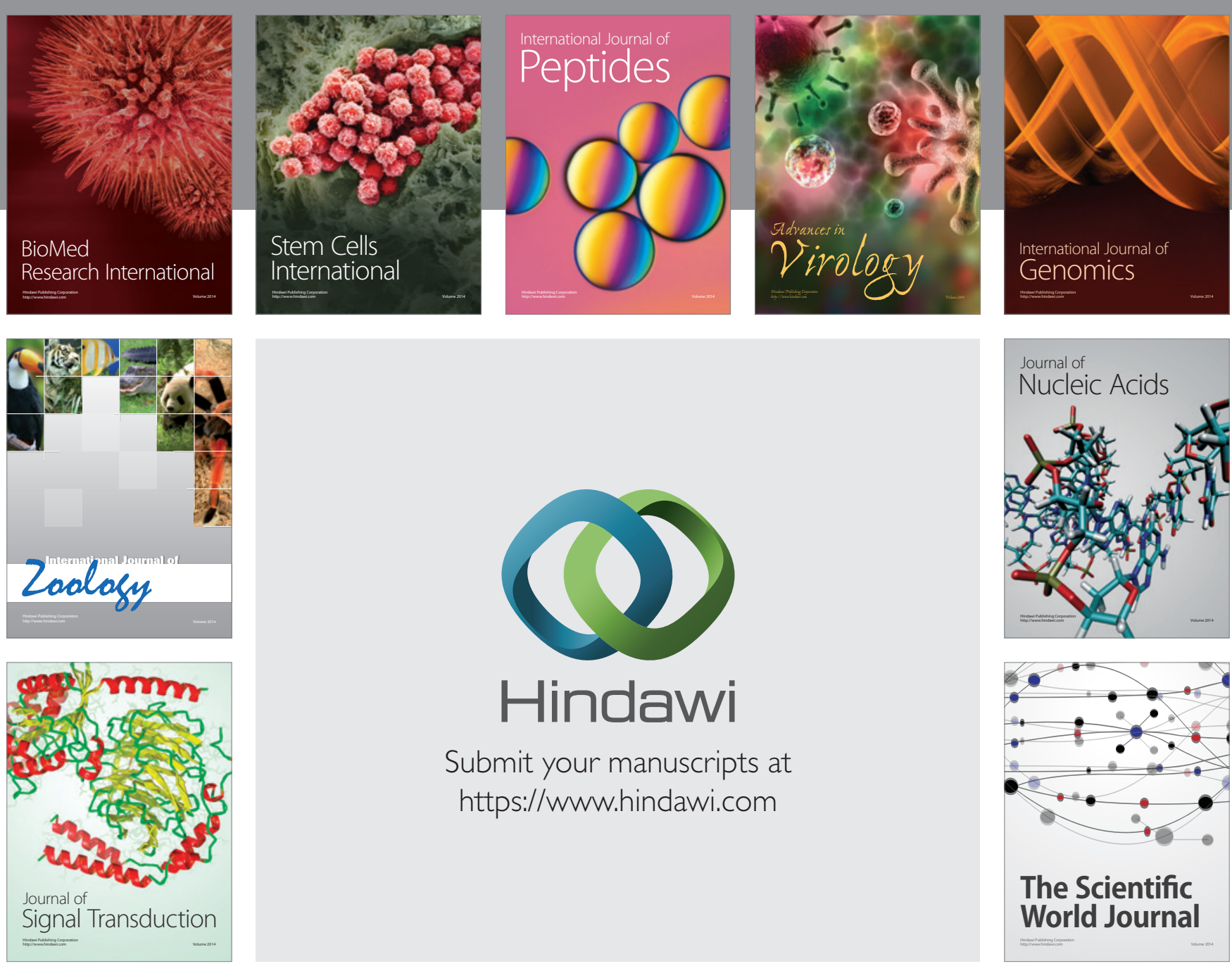

Submit your manuscripts at

https://www.hindawi.com
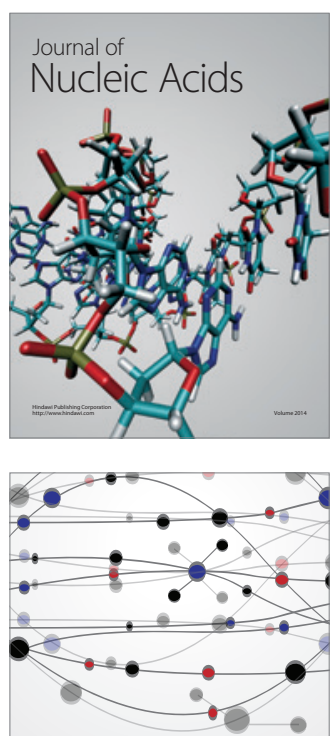

The Scientific World Journal
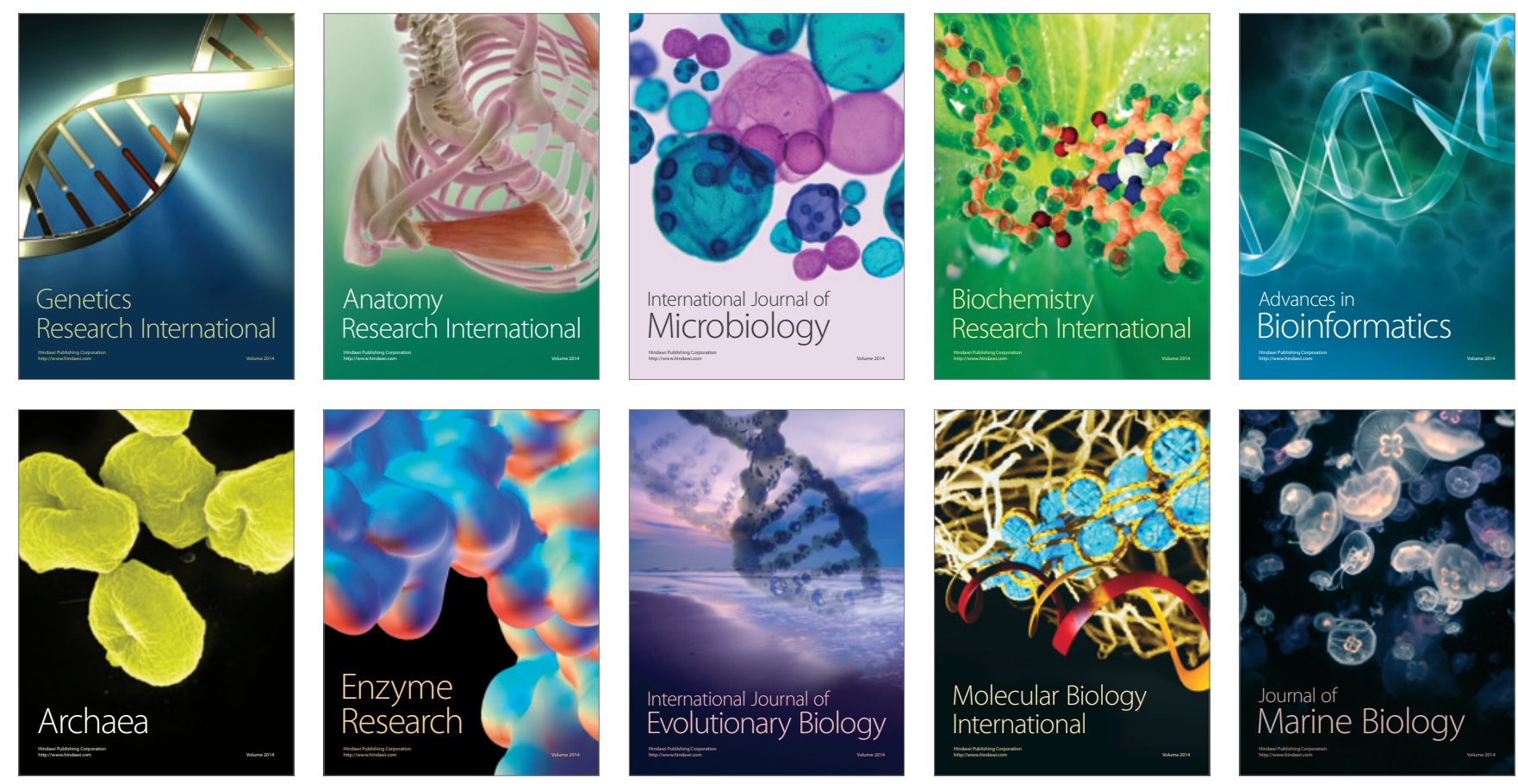